\title{
Design of the Landslide Multi-factor Monitoring System Based on the GNSS Technology
}

\author{
Meng Qingjia ${ }^{1, *}$, Wang Chenhui ${ }^{1}$, and Guo $\mathrm{Wei}^{1}$ \\ ${ }^{1}$ Center for Hydrogeology and Environmental Geology Survey, CGS, 1305 Qiyi Zhong Road Baoding, China
}

\begin{abstract}
Based on the multi-element monitoring of landslide, this paper adopts the GNSS technology to build the three-dimensional space monitoring system to research the landslide multi-element threedimensional space monitoring technology. Through collecting the rainfall, soil moisture content, slope, pore water pressure, stress, single point surface deformation etc, the system has many key technologies such as multi-parameter, information acquisition, data fusion analysis and real-time early warning of landslides and integrate various elements by using the professional technology. This research provides an important reference for the landslide disaster prevention.
\end{abstract}

\section{Introduction}

There are many problems in geological hazards, such as the complex process of disaster pregnancies and disastercausing patterns, the difficulty of early identification and monitoring and early warning, and insufficient technical support for risk prevention. Therefore, the national disaster prevention and mitigation strategy has a great demand for the technical equipment of geological hazard monitoring. The key technology of three-dimensional space monitoring can integrate the monitoring technology of rainfall, soil water content, tilt, pore water pressure, stress and other landslide factors, and realize the integration of professional monitoring technology system. In this technical system, the multiple information processing at each monitoring point is transformed into a time series problem. By using data fusion technology, a set of comprehensive information is synthesized, and the landslide is monitored and predicted directly from point to surface ${ }^{[1]}$. The main task of landslide monitoring is to grasp the characteristic information of landslide deformation and failure by combining deformation monitoring and induced factors monitoring, analyze the dynamic law, and realize the reliability and validity of monitoring in space, time and scale $^{[2]}$.

Based on the key technologies of the landslide multidimensional and multi-parameter information acquisition about rainfall, soil moisture content, tilt, pore water pressure, stress and others, data fusion analysis and realtime disaster warning, this paper carries out the research of landslide multi-factor three-dimensional spatial monitoring technology. This research is helpful toimprove the application effect of geological hazard monitoring technology and provide technical support for geological hazard monitoring and early warning.

\section{Overall design of the monitoring system}

The integration of the high-precision three-dimensional spatial positioning technology and multi-factor landslide real-time monitoring technology can increase the spatial dimension information of monitoring data on the basis of the original time dimension. This integration effectively improve the effective information quantity and use value of monitoring data. In addition, through multi-node deployment, the original monitoring mode of point monitoring can be extended to the overall monitoring. Through data fusion analysis technology, a large number of basic monitoring data are obtained, such as rainfall, soil moisture content, slope, single point surface deformation, stress and other key elements of landslide, as well as three-dimensional coordinates of monitoring points, and used to analyze and obtain the overall threedimensional spatial deformation of landslide. The above data fusion analysis technology, combined with the location of monitoring points and other means, can be simple and efficient to achieve the overall control of the landslide condition. In addition, the representation of the spatial location makes the original monitoring parameters have the three-dimensional space vector attributes. This is of great significance for data mining and analysis, which can improve the value of application scenarios such as water, stress, soil moisture transmission direction and speed research.

\subsection{High Precision Three-Dimensional Space Positioning Technology}

Based on the basic data of three-dimensional coordinates, velocity and precise timing provided by GNSS, a low-

Corresponding author: menmen-00@163.com 
power and high-reliability satellite signal receiving device suitable for geological hazard monitoring scenarios is developed to realize the functions of receiving, filtering, storing and transmitting satellite signals. Research high-precision three-dimensional space positioning technology and related algorithms, research three-dimensional space position calculation algorithm and implementation method based on GNSS, carry out static RTK, dynamic RTK, multi-satellite and multisource data joint algorithm research and compile related software. By means of algorithm analysis, error propagation mechanism analysis, real scene testing and other means, the performance and effect of the algorithm are continuously improved, and finally the positioning accuracy of millimeter level is achieved. According to the satellite trajectory and the characteristics of satellite signal, the antenna and hardware structure of the receiving device are optimized. According to the actual needs of disaster monitoring, the working mode, positioning accuracy, calculation frequency, power consumption and volume are considered and coordinated. Through collecting monitoring data of GNSS landslide deformation under monitoring point environment, the observation range, signal-to-noise ratio and multi-path effect sequence of different environmental satellites are analyzed and corrected ${ }^{[3]}$. Key technologies such as realtime cycle slip detection, fast ambiguity fixing and multi-system joint positioning are adopted to eliminate system errors under short baseline and medium baseline conditions to achieve high-precision positioning ${ }^{[4]}$.

\subsection{Multi-factor Real-time Monitoring Technology}

The occurrence of landslides is not only related to topographic and geological conditions, but also to the inducing factors of geological conditions. According to the main characteristics of landslide, the related factors are summarized: rainfall, stress, slope, single point surface deformation, soil moisture content, etc ${ }^{[5]}$. Landslides are time series events occurring at different stages of development. Single factor monitoring landslide, such as displacement monitoring, is often aimed at local monitoring, which can't fully reflect the dynamic change of the overall landslide. This system integrates many factors, takes the key related factors of high-level long-distance landslide and actual monitoring needs as the guidance, integrates the corresponding monitoring variables, and gradually realizes from quantitative analysis to qualitative analysis..

\subsection{Data fusion technology}

Landslide monitoring multi-factor system composed of Multi-heterogeneous sensors involves data determination, data management complexity processing and evaluation of multiple models in data fusion. The basic combination model is established to comprehensively analyze many influencing factors of landslide deformation, so as to improve the possibility of landslide prediction. In this model, the artificial neural network model is used as input variable, and the genetic algorithm model is combined with GM grey prediction model ${ }^{[6-7]}$.

The system integrates high-precision threedimensional space positioning technology and multifactor real-time monitoring technology from hardware and software levels to form a unified monitoring equipment with strong practicability, stability and reliability. Through embedded software control and buslevel data exchange, the system realizes high-speed fusion and calculating of multi-factor real-time monitoring data and three-dimensional spatial positioning data, so as to obtain real-time dynamic data of deformation speed and acceleration on the basis of basic monitoring data.

\section{Hardware Circuit Design of Monitoring Equipment}

The hardware design of monitoring equipment adopts the idea of generation by generation optimization. In the initial stage, hardware design was carried out in the way of independent functional modules and unified baseboard. In the design of functional modules, priority is given to the realization of functions and the effect of implementation. In the design of the unified baseboard, it first meets the operational requirements of each functional module and the realization of data fusion. In terms of hardware resources, more abundant configuration is adopted to ensure that monitoring equipment has all the functions and requirements required by the project.

The basic components of the hardware: highprecision three-dimensional positioning module, multielement real-time monitoring module, equipment infrastructure board

\subsection{High-precision three-dimensional positioning module}

The module mainly performs data interpretation, analysis and processing on monitoring data. The module mainly includes an antenna, a clock, a pseudo random code generator, a digital filter circuit, and a signal analysis processor.

\subsection{Multi-element real-time monitoring module}

This module is responsible for front-end data acquisition. The module is connected to a variety of sensors (the rainfall, soil moisture content, slope, pore water pressure, stress, single point surface deformation etc.), and the data is packaged and sent to the cloud. The module mainly includes an $\mathrm{AD}$ conversion circuit, an analog signal conditioning circuit, a 485 serial port circuit, and a general-purpose interface.

\subsection{Equipment infrastructure board}

This module mainly covers power supply, data storage, remote communication and embedded processing. The 
module is mainly composed of an embedded processor, a power supply module, a local storage circuit, a near field/far field communication circuit, and a clock circuit.

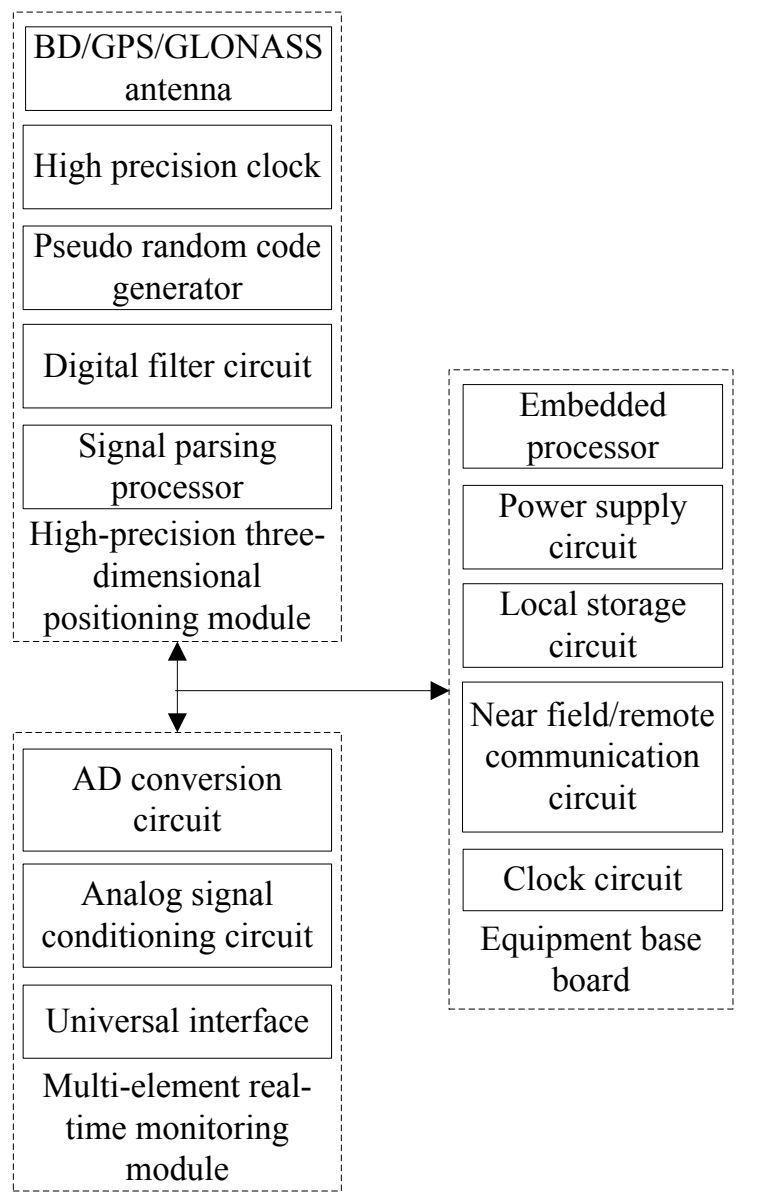

Fig. 1. Hardware system design.

\section{Research on Three-Dimensional Space Location Algorithms}

GNSS is gradually used in high-precision deformation monitoring and analysis, and the accuracy can reach millimeter level ${ }^{[8]}$.In the three-dimensional space location algorithm, the ambiguity resolution method and the normal equation superposition method are used. Fuzzy fixing is carried out by $\mathrm{Z}$ transformation matrix construction method and decision function, and the fuzziness is analyzed and screened comprehensively. Pseudo-range phase combination combined cycle slip detection is used to reduce the number of cycle slip combination, and short-range and medium-range GNSS baseline is used to monitor landslide deformation. ${ }^{4]}$

The important stage of the design of the program is the processing of monitoring data. This system first imports the monitoring data into the database for preprocessing. In the next step, the data is shunted, repaired, calculated, and standardized. The key problem of the design is to analyze and solve the satellite positioning data, model correction, floating point solution, fixed ambiguity, and baseline settlement. The data solving process is based on regional network adjustment and time series analysis. The settlement method removes the possibility of error in the data. Eventually, the final amount of deformation of the landslide object is obtained.

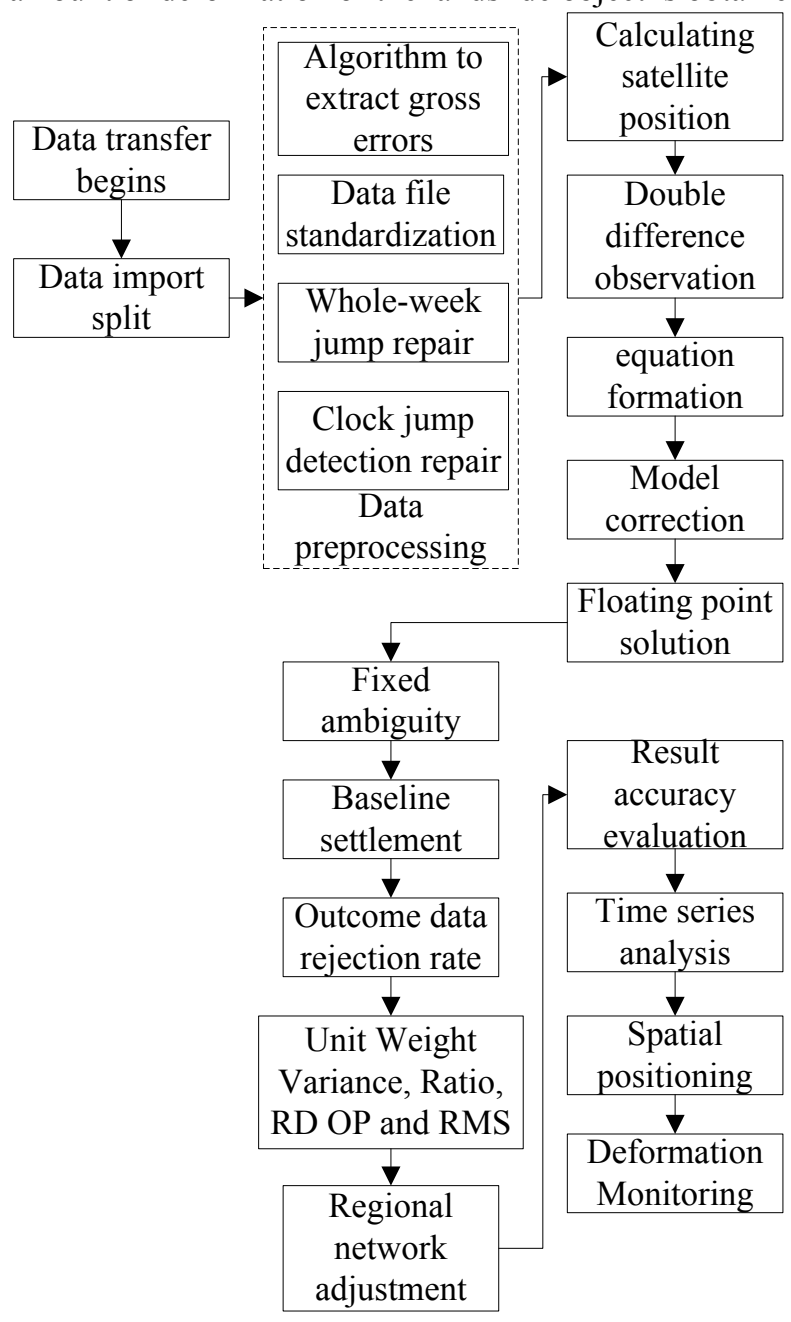

Fig. 2. GNSS monitoring system work flow chart.

\section{Conclusion}

The landslide multi-factor monitoring system based on GNSS technology integrates the collected monitoring data, satellite remote sensing data, geological structure model of disaster body and standardized early warning analysis model to form an efficient, reliable and stable intelligent command and dispatch management system. Through the algorithm analysis and trend discrimination of the system, the automatic data report forms of periodic, periodic and unexpected events can be realized. The system can provide effective data decision analysis and technical support for landslide monitoring.

This research is supported by the development of intelligent monitoring equipment for key elements of landslides in Karst Mountains (2018YFC150480502), and also supported by geological disaster monitoring technology integration and application projects.

\section{References}

1. Guo Ke, Peng Jibing, Xu Qiang, Realization of the extraction of comprehensive information of multi- 
station monitoring data of landslide with the technique of multi-sensor target tracking, PROGRESS IN GEOPHYSICS, 20, 808-813 (2005)

2. Liu Huan, Chen Hai, Application Research of Integrated Monitoring Technology in Landslide Prediction and Forecas, Transportation Science \& Technology, 3, 10-12 (2018)

3. Han Junqiang, Huang Guanwne, Li Zhe, Multipath Effect analysis and Processing Method of GNSS Landslide monitoring Under Complicated environment, Journal of EarthSciences and Environment, 40, 355-362(2018)

4. Xiao Yugang, Jiang Weiping, Chen Hua, Research and Realization of Deformation Monitoring Algorithm with Millimeter Level Precision Based on BeiDou Navigation Satellite System, Acta Geodaetica et Gartographica Sinica, 45, 16-21(2016)
5. Wan Ling Chuan, GNSS Real-Time Relative Positioning Technology and its Application in Landslide Monitoring, Chang'an University, 2050(2018)

6. Wang chunhua, Appication of complex event processing to landslide monitoring sensor data integration research, East China Normal University, 15-20(2016)

7. Fan Junqing, Research on multi-source heterogeneous sensor information fusion for landslide monitoring, China University of Geosciences, 20-25(2015)

8. Sun Bo, Method research on comprehensive Treatment and Data Fusion of Multiple Monitoring Data, China University of Geosciences, 19-27(2012) 